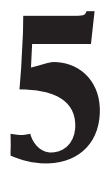

\title{
UN JUEGO DE ESPEJOS: PENSAR LA DIFERENCIA DESDE LA PEDAGOGÍA INTERCULTURAL
}

\author{
(A GAME OF MIRRORS: THINK THE DIFFERENCE FROM THE \\ INTERCULTURAL PEDAGOGY)
}

Eduardo S. Vila Merino

Universidad de Málaga

\section{RESUMEN}

Con este artículo se pretenden aportar reflexiones que nos permitan pensar las diferencias que nos constituyen desde el tamiz intercultural para, a partir de su consideración, ver otras maneras de entender las relaciones educativas bajo los principios de la equidad y la inclusión. Para ello, en primer lugar se desgrana el concepto de diferencia en consonancia con el de identidad, de forma que obtengamos un sustrato teórico desde el que elevar nuestros análisis y propuestas. En segundo lugar se establecen qué principios se derivan de la anterior conceptualización desde la perspectiva intercultural y de cara a la atención a la diversidad desde el punto de vista educativo. Finalmente, se lleva todo esto a una perspectiva en forma de capacidades desde la cual le pongamos sentido aplicado a esos principios como competencias docentes necesarias para abordar en los espacios educativos los planteamientos desarrollados.

\begin{abstract}
With this article we try to contribute with some reflections that permit us to analyse the differences that constitute us from the intercultural sieve. From this point, we aim to develop other ways of understanding the educational relations under the principles of equity and inclusion. In order to achieve such aims we firstly analyse the concept of difference in relation to that of identity, so that we obtain a theoretical substratum from which to raise our analyses
\end{abstract}


and proposals. Secondly, we establish what principles stem from the previous conceptualization from the intercultural perspective and with a view to the attention to the diversity from the educational point of view. Finally, we seek competences perspective in relation to which apply this principles understood as necessary teachers competences to undertake the developed concepts in the educational dimensions.

\section{INTRODUCCIÓN}

«Siempre fuiste mi espejo. Quiero decir que para verme tenía que mirarte.» Estas palabras de Julio Cortázar nos llevan al resbaladizo territorio de la identidad y de la diferencia, al intrincado laberinto de la educación en y para la diversidad, a las múltiples cartografías sociales de lo que pensamos desde la alteridad que nos constituye y, sobre todo, al rostro de quien es en la medida que hay otro u otra que le ayuda a ser desde el juego de espejos que constituyen las relaciones humanas.

Estas páginas pretenden aportar reflexiones que nos permitan pensar las diferencias que nos constituyen desde el tamiz intercultural para, a partir de su consideración, ver otras maneras de entender las relaciones educativas bajo los principios de la equidad y la inclusión. Para ello en primer lugar se desgrana en concepto de diferencia en consonancia con el de identidad, de forma que obtengamos un sustrato teórico desde el que elevar nuestros análisis y propuestas. En segundo lugar se establecen principios vinculados con la anterior conceptualización desde la perspectiva intercultural y de cara a la atención a la diversidad desde el punto de vista educativo. Finalmente, se lleva todo esto a una perspectiva en forma de capacidades desde la cual le pongamos sentido aplicado a esos principios como competencias docentes necesarias para abordar en diversos espacios educativos los planteamientos desarrollados.

\section{IDENTIDAD Y DIFERENCIA}

Hablar de identidad es hacerlo de un proceso complejo y con múltiples factores en interacción de índole personal, social y cultural, lo cual otorga un papel destacado en su configuración a la educación como elemento clave para la constitución de la subjetividad, la intersubjetividad, las diferencias y la organización identitaria.

Dado el entramado que supone siquiera un esbozo de definición de la identidad, como aproximación a la misma debemos tener en cuenta múltiples elementos. En todo caso, siguiendo a Bilbeny (2002), resulta prioritario 
destacar que las identidades no son productos acabados, sino que están continuamente haciéndose, teniendo un carácter cualitativo y autorreferente a la vez que con ello permite ver la alteridad ajena. Esto significa que tanto las identidades como las diferentes no tienen un carácter homogéneo ni aislado, sino que permanentemente contactan con otras y se establecen apropiaciones mutuas, e incluso en el contacto dialéctico consigo mismas en su contexto pueden desproveerse de lo propio para seguir siendo ellas mismas, pero de otro modo. Por ello debemos enfatizar el carácter también contingente de las identidades, lo que conlleva que los fenómenos de apropiación, desposeimiento y reapropiación que caracterizan la relación, no la sustancia, que es la identidad, sean fenómenos siempre selectivos y culturales, no mecanizados, y que se construyen a su vez desde las propias diferencias.

Por el contrario, los procesos de globalización parecen haber hegemonizado una creencia contraria sobre la naturaleza de la identidad, puesto que en lugar de aceptar su carácter fundamentalmente híbrido, resultado y a la vez posibilidad de diversos cruces de experiencias, ha exagerado su naturaleza particular y sus funciones individualizadoras, motivando una cierta confusión conceptual entre identidad y etnicidad, al mismo tiempo que invisibilizando, o al menos llevándolas a un terreno secundario, a otras formas de identidad como las vinculadas al género, negando o discriminando en función de las diferencias. Todo ello haciendo énfasis en que la pluralidad de identidades y culturas no es algo que constituya ningún problema, y si los genera es en todo caso por la manera en que dicha pluralidad es tratada social o institucionalmente, transformando las diferencias en desigualdades o 'pretextos' para la exclusión.

De lo anterior podemos deducir que la construcción de la identidad es un proceso, pero no una secuencia definida, sino que tiene un carácter dialéctico y se configura a través de distintas dimensiones desde la práctica social (Jenkins (1996) habla de «momentos de identificación.») Así, podemos considerar que la identidad se basa en normas de pertenencia fundadas sobre oposiciones simbólicas, entendiendo esas pertenencias como espacios de experiencia y sentido que van configurando desde su multiplicidad el ahora de lo que somos (Bárcena, 2004), estando ese ahora ligado de forma indisoluble al de un otro/a también plural y diverso. Por eso mismo no debe entenderse esto de forma restringida ni rígida, porque ello conlleva un riesgo enfatizado desde el punto de vista educativo, donde los ejercicios reduccionistas y los juicios dicotómicos no suelen beneficiar la diversidad al minimizar la complejidad de los procesos, como los de construcción identitaria. Por tanto, las identificaciones entre las nociones de identidad, diferencia y cultura constituyen un riesgo para el pensamiento pedagógico al intentar reducir a los individuos a quienes se dirige a conceptos o categorías abstractas, 
privándolos de su corporeidad, sus experiencias y sus voces, por lo que sería necesario que dichas identificaciones se den dentro de un proceso heterogéneo y de apertura al otro u otra, no encorsetado en su prejuicio.

Desde aquí que a menudo se haya hablado de los procesos de identificación como laberintos, pero unos laberintos donde, se insiste, el binomio identidad-diferencia cobra su sentido desde la existencia y la presencia del otro o la otra. Por tanto, las diferencias son generadoras de identidad, y éstas a su vez remiten a la diversidad humana y su papel fundamental para una concepción ética de nuestro ser-en-el-mundo, por lo que no debe ser siquiera insinuada cualquier manifestación de opresión, alienación o discriminación al respecto. Se trata también, en cierto sentido, de modificar el concepto moderno de experiencia, excesivamente centrada en el sujeto, por una experiencia entendida en términos de confrontación con lo otro y con los otros/as (Innerarity, 2001), donde el ser identitario genera y es generado por la propia alteridad, concretándose en la unidad identidad-diferencia, a partir de la cual «no sólo la identidad es inmediatamente el momento de la reflexión en sí, sino que asimismo lo es la diferencia - diferencia en sí, diferencia reflejada - Ésta, en tanto que tales momentos suyos son reflexiones en sí, es diversidad» (Gabilondo, 2001, 63).

Todo esto nos lleva a considerar que en el contexto actual se están generando nuevas formas de construcción de la identidad, basadas en la interrelación dialéctica entre lo colectivo y lo individual, desde una vertiente política donde, a la reconstrucción de las identidades particulares que la inmersión en una sociedad de pluralidad compleja lleva consigo, hay que añadirle lo imprescindible que es contar con una nueva forma de identidad colectiva que se construya a partir de las anteriores a la vez que las trascienda (Pérez Tapias, 2007). De esta manera, podemos inferir que para la configuración del binomio identidad-diferencia debemos tener presente que el mismo requiere de un sustrato cultural y que la cultura necesita permanentemente de traducciones, por lo que entender quién traduce, a quién, a través de qué significados, etc., se torna fundamental. De hecho, las diferencias culturales surgen en el marco de la diversidad de interacciones entre personas y colectivos, lo que ofrece una visión dinámica de la cultura ajena a la integración por asimilación y a la configuración de estereotipos como modus operandi de tratamiento de las diferencias, así como promotora de la asunción del individuo como agente activo en la construcción cultural. Esta cuestión nos lleva a una consideración mayor, y es que «inferir la identidad desde la cultura significa negar la complejidad respecto de las formas sociales de intercambio de los individuos y los grupos como elementos constitutivos de lo cultural» (Marí Ytarte, 2007, 49. En este sentido podemos afirmar, a modo de corolario, que las identidades (en plural) se configuran a partir de las diferencias (también en plural) entre distintas culturas en in- 
teracción, lo que nos hace ver su complejidad sustantiva a la vez que nos desvela su potencialidad para la construcción social y educativa, la cual consideramos fundamental desarrollar a través del paradigma intercultural, el cual se ha visto bastante vapuleado, tergiversado a veces, encorsetado otras, o simplemente vaciado de contenido utilizando el término para cuestiones que poco o nada tienen que ver con su verdadero sentido.

\section{LA PERSPECTIVA INTERCULTURAL COMO EJE DE LA REFLEXIÓN IDENTIDAD-DIFERENCIA.}

La reflexión sobre la educación en la actualidad no puede realizarse con un mínimo de rigurosidad y honestidad intelectual desligada de la interculturalidad como referente. Esto es así porque ni la escuela actual, como valuarte de la educación formal, ni el amplio abanico de teorías y prácticas de la educación social, puede dejar de vincularse a la diversidad y los derechos inherentes a esa presencia que los otros y otras diferentes tienen en ella, dotándola de sentido y potencialidad transformadora. Lo anterior nos lleva, entre otras cuestiones en el ámbito de la multiculturalidad, a plantearnos la necesidad de repensar la pedagogía desde una valoración de la diversidad cultural que no la convierta en diferenciación cultural ni desigualdad social, a la manera que plantea Bhabha:

«La idea de que la diversidad cultural constituye un problema porque ya hay muchas culturas diferentes no es la razón por la que existe la diferencia cultural. La diferencia cultural es un discurso elaborado en un momento en el que se están desafiando elementos del poder o la autoridad (...) La diferencia cultural no resulta difícil, si se quiere, por el hecho de que haya diferentes culturas, sino porque existe una cuestión particular sobre la redistribución de los bienes entre las culturas, o los fondos de las culturas, o el surgimiento de minorías o inmigrantes en una situación de recursos -a dónde deben dirigirse estos- o la construcción de escuelas y la decisión sobre si la escuela será bilingüe, trilingüe o lo que deba ser. Es en este punto en el que se produce el problema de la diferencia cultural. De este modo, constituye verdaderamente un argumento contra la naturalización de la idea de cultura» (Bhabha, cit. Giroux, 2001, 71).

Esto nos debe hacer sentar las bases para una concepción pedagógica intercultural que dé respuesta a nuestras realidades multiculturales y los desafíos de la globalización, desgajada del corsé de su definición neoliberal y tristemente hegemónica. Se trata de avanzar hacia una comprensión de la multiculturalidad como interpretación ampliada de la democracia, trascendiendo el multiculturalismo liberal y tolerante que habla retóricamente de lo Otro pero sin su Otredad (Martínez de Bringas, 2007). En este sentido, hay que re- 
cordar que las estrategias multiculturalistas suelen rechazar el reconocimiento de los espacios de confluencia en la coexistencia (multicultural) como paso previo para una convivencia (intercultural) entre diferentes comunidades en el seno de una sociedad concreta. E incluso yendo más allá, podemos afirmar con Zizëk $(1998,114)$ que: «La insistencia en el multiculturalismo entendido como la coexistencia híbrida y mutuamente intraducible de diversos mundos de vida culturales puede interpretarse también sintomáticamente como la forma negativa de la emergencia de su opuesto, de la presencia masiva del capitalismo como sistema mundial universal.»

Por lo tanto, además de lo dicho, podemos distinguir estas cuestiones respecto a las diferencias entre multiculturalidad e interculturalidad (Marí Ytarte, 2007, 84-86):

a) La multicultura se refiere a una situación de hecho, la de la coexistencia (y convivencia) de grupos culturales diferentes en un mismo territorio, y la intercultural a una estrategia de acción, la de conseguir que esos grupos culturales se nutran mutuamente de sus diferencias al tiempo que crean, o comparten, proyectos de vida en común.

b) Lo multicultural correspondería a un discurso que acentúa el valor de las diferencias culturales, miembros que lo intercultural privilegiaría el principio de semejanza y la noción de igualdad.

c) La distinción esencial estaría en la limitación conceptual del prefijo multi-, ya que no implica 'comunicación' e intercambio entre grupos culturales. Lo multi- tendría un carácter descriptivo y el inter- más normativo.

La interculturalidad aquí, lejos de verse como una categoría abstracta, racional y homogénea, remite más a la confrontación y entrelazamiento polémico de diferentes grupos, comunidades e identidades que entran en conexión e intercambio. Así, mientras que multiculturalidad liberal supone la aceptación teórica de lo heterogéneo; la interculturalidad, en un buceo más profundo y delicado, evidencia que las diferencias son lo que son y se afirman identitariamente a partir de intensas relaciones de negociación y de dialécticas conflictivas desde los cuales podemos intentar desarrollar préstamos recíprocos de significados para la construcción intercultural. Con esto es necesario aclarar también que la interculturalidad tampoco es un tránsito de las diferencias a las fusiones que las diluyen, puesto que los flujos presentes en las realidades multiculturales son conflictivos y hay que saber ubicarse en ellos reconociendo las diferencias desde la lógica de los procesos de producción de condiciones de igualdad/desigualdad, conexión/desconexión, inclusión/exclusión (García Canclini, 2005) como referentes claros a la 
hora de abordar la construcción intercultural. Por lo tanto, el discurso intercultural se interrogaría entonces por una doble dimensión de la cultura y por la posibilidad de su articulación conjunta: como proceso de multiplicidad desde lo común y como forma de expresión de las pertenencias subjetivas, individuales y colectivas en tanto que reconocimiento de lo particular. Y a su vez, ese discurso intercultural requiere por parte de la pedagogía de una interrogación, de un modo de preguntarse sobre los modos de articular el propio discurso sobre la cultura y las diferencias (Marí Ytarte, 2007).

Desde el ámbito educativo, por tanto, el proceso intercultural está íntimamente ligado a la perspectiva de los derechos humanos y nos plantea como exigencia ética la búsqueda de códigos y puentes de conexión comunes y recíprocos a todas las identidades y cosmovisiones en interacción y/o conflicto, de manera que éstos nos sirvan para el contraste y crítica de los mecanismos culturales presentes y, a partir de ahí, para establecer pautas de traducción plausibles desde las distintas ópticas culturales implicadas como condición de posibilidad para la convivencia. De esta forma, la presencia y la relación con el otro u otra y su otredad nos puede ayudar a transformar el 'choque de civilizaciones' en un 'encuentro de vecinos' (Bauman, 2007). Pero eso sí, la propia interculturalidad debe aquí asumir la condición inconmensurable de ciertos enunciados culturales, los cuales no es posible codificar en un lenguaje de mediación intercultural en determinados contextos. Se trata entonces de contrastar la gramática de la interculturalidad asumiendo la dificultad y las limitaciones del propio proceso intercultural cuando las condiciones del conflicto cultural lo hacen insensible a la traducción. Ahí es donde la interculturalidad debe resituarse como elemento fundamental para el acceso a un posible pacto cultural que recree las condiciones para la convivencia. Por eso se hace necesario para la interculturalidad el llevar a cabo análisis de los procesos de las diferencias y las causas estructurales que generan dinámicas de desigualdad en recursos y capacidades en contextos concretos, de falta de reconocimiento para determinadas identidades personales y/o colectivas, de desconexión selectiva de posibilidades comunicacionales y de bienestar que lleva a la exclusión. Ello exige evitar una construcción meramente victimista de los/las excluidos/as-oprimidos/as, perversión tan propia del multiculturalismo liberal, y superar una comprensión esencialista y dogmática de la alteridad que la aleja del sujeto (Martínez de Bringas, 2007). Y esto a su vez conlleva que hablemos de la pedagogía intercultural como una pedagogía de la escucha, de los encuentros y de las posibilidades, de las negociaciones y los espacios comunes, de las indefiniciones y las pertenencias, de lo afianzado y aquello por construir.

En este sentido podemos hablar de la necesidad, en el marco de la gestión de las diferencias, del «imperativo intercultural»(Pérez Tapias, 2007, 152) «como imperativo moral que obliga a romper los monólogos etnocén- 
tricos para dar paso a un diálogo cuya multiplicidad de voces anuncie la 'polifonía de los pueblos' (...) Esto no será posible si no tomamos en serio la obligación moral de activar el diálogo entre los culturalmente diferentes, abriéndose cada cual a la alteridad diversa, para tratar de lograr los necesarios acuerdos sobre las cuestiones de justicia que hemos de resolver para hacer viable la convivencia.» Y para ello hay que renunciar a cualquier pretensión de lugar privilegiado en la esfera relacional, lo cual incluye una reformulación de las relaciones de poder que en el marco intercultural se ven afectadas por elementos de diálogo y traducción bajo el eje común de la búsqueda de justicia en términos de igualdad real de condiciones, exigiendo por un lado la igualdad social y de derechos desde las diferencias culturales y por otro el reconocimiento y participación de las personas y colectivos cuya identidad cultural se diferencie de la hegemónica (García y Goenechea, 2009).

Por todo ello, la perspectiva intercultural pretende denunciar y trascender modelos históricamente hegemónicos de gestión de la diferencia para visualizar espacios de confrontación y conflicto donde, sin caer en el relativismo, aceptemos la relatividad contingente a la interacción cultural como eje de la acción educativa desde la mirada al otro/a cultural (Vila Merino, 2007). Todo ello sin obviar la existencia de condicionantes culturales y políticos, en forma de voluntades reales más allá de los discursos, que limitan la implicación e implantación efectiva de la educación intercultural, y que van desde la propia formación docente a cuestiones organizativas, de gestión de recursos, planteamientos curriculares etnocéntricos, etc.

Esto implica una manera dialógica de ver las relaciones de forma que se posibiliten espacios públicos compartidos y confluencias desde la concreción del 'espacio moral' entre culturas que supone la interculturalidad y sus consecuencias pedagógicas, lo cual conlleva un planteamiento diferente de la interacción con las culturas minoritarias, más allá de lo conocido como integración, que resulta un concepto fallido y poco apropiado en términos interculturales. En palabras de Pérez Tapias (2007, 61): «Este término, incluso manejado con la mejor de las voluntades, aún lleva consigo demasiadas connotaciones asimilacionistas y, por tanto, el riesgo de imponer a los diferentes exigencias particularistas no justificables como universales. Por ello, mejor que hablar de integración, aquilatando más en torno a lo común que ha de suscribirse, por responder efectivamente a los principios universalistas de la democracia en los que podemos reconocer una validez transcultural, lo pertinente es hablar de inclusión.» La lógica de la inclusión, y no de la integración, es la que tiene un verdadero sentido intercultural, de la forma que vamos articulándolo, y desde ahí vamos a adentrarnos en el mar de la educación intercultural como referente teórico y las competencias docentes interculturales necesarias como capacidades a desarrollar en y desde el profesorado. 


\section{LAS COMPETENCIAS INTERCULTURALES: CAPACIDADES DOCENTES NECESARIAS PARA EL DESARROLLO DE LA EDUCACIÓN INTERCULTURAL}

Todo lo anterior, indudablemente, supone un desafío para la educación en general y para la configuración de la manera de entender la gestión de las diferencias en el ámbito de la educación intercultural, lo cual es en parte reflejado por Bruner (1997) en el prólogo de su libro 'La educación, puerta de la cultura':

«La tarea de las nuevas generaciones es aprender a vivir no sólo en el amplio mundo de una tecnología cambiante y de un flujo constante de información, sino ser capaces al mismo tiempo de mantener y refrescar también nuestras identidades locales. El desafío es poder desarrollar un concepto de nosotros mismos como ciudadanos del mundo y, simultáneamente, conservar nuestra identidad local como mexicanos, zapotecos, españoles o catalanes. Posiblemente tal desafío representa para las escuelas, y la educación en general, una carga como nunca en la historia.»

Esta responsabilidad implica que educativamente debemos tener muy presente de qué hablamos cuando lo hacemos de educación intercultural, así como de la necesidad de replantearnos permanentemente nuestra concepción de diversidad cultural y el tratamiento que hacemos de las diferencias desde un punto de vista social, político y pedagógico. De hecho, la pedagogía ha sido uno de los ámbitos en los que las teorías interculturales han tenido una mayor importancia y desarrollo, naciendo con la pretensión de dar respuestas a la relación identidad-diferencia y su atención en el marco educativo, en aras de conseguir que dichas relaciones sean cada vez más igualitarias y basadas en criterios de justicia social. Y para su puesta en práctica resulta imprescindible no caer en simplificaciones caducas o segregadoras, pues a veces podemos caer en el error de tratar la cuestión intercultural como algo homogéneo, descontextualizado y con pocas complejidades semánticas, y la realidad se empecina en mostrarnos lo contrario, ya hablemos desde las reivindicaciones de las minorías étnicas, la revitalización de culturas después de procesos de descolonización, las migraciones sean por motivos políticos, económicos, sociales o personales, los flujos informativos que transportan los medios de comunicación, la diversidad física o sensorial, el intercambio cultural, etc.

En este sentido, compartiendo las ideas de Touriñán $(2008,37)$, podemos diferenciar un objetivo de la educación intercultural si la entendemos como tarea y otro si la entendemos como resultado. En el primer caso, hablaremos del desarrollo de destrezas, hábitos, actitudes y conocimientos que capacitan a las personas para estar, moverse, intervenir, actuar, aprender 
e interrelacionarse con la diversidad cultural, porque de lo que se trata en la tarea es de construir experiencia axiológica sobre mi identidad, la del otro, la diversidad y la diferencia. En el segundo caso, nos referiremos a la adquisición en el proceso educativo de un conjunto de competencias que capacitan al educando para decidir y realizar su proyecto, utilizando la experiencia axiológica intercultural. Por eso tiene sentido hablar de la educación intercultural como uso y construcción de experiencia axiológica sobre la identidad y la diferencia, para construirse a uno mismo y reconocerse con el otro en un entorno cultural diverso de interacción.

Siguiendo esta tesitura, vemos cómo la pedagogía intercultural ha solido estructurarse a través de dos grandes líneas de actuación: como atención educativa a personas inmigrantes, minorías o los 'otros culturales' y como prevención y educación antirracista. Esto ha provocado que se haya visto a la educación como un elemento clave para dar respuesta a los conflictos derivados de las diferencias y las desigualdades, de los ámbitos identitarios y de socialización, de la exclusión y los intentos de 'integración' entendida como homogeneización, lo cual hace que inclusión, participación e interculturalidad sean tres dimensiones indisolubles de la fenomenología social y educativa a la que no puede obviarse ene todo análisis pedagógico riguroso.

En un mundo globalizado y basado en las interacciones, más o menos asimétricas, la reflexión y los cuestionamientos de la perspectiva intercultural en torno a las identidades y las diferencias, a la diversidad cultural y las pertenencias y adscripciones sociales, supone un bálsamo teórico necesario para la pedagogía intercultural como estrategia para la puesta en práctica de la diferencia y hacia la misma. Pero esto debe hacerse sin confundir el objeto de dicha pedagogía intercultural, que no serían las personas 'inmigrantes' o los 'diferentes culturales', sino los propios sistemas e instituciones educativas, los espacios y discursos pedagógicos, puesto que desde los mismos se configuran las identidades y formas de socialización y pertenencia aludidos.

En todo caso, la construcción de una sociedad intercultural conlleva articular una serie de procesos de cambio que lleven a una definición del mismo al margen de limitaciones de corte nacionalista o excluyente, ajena al etnocentrismo y que facilite el intercambio y la toma de referencia del estilo de vida cívico desde una perspectiva de derechos. La capacitación educativa desde el ámbito intercultural debe suponer tanto la implicación política y social en orden a realizar aquellas transformaciones que acaben con las desigualdades enajenadas injustamente desde la diferencia, como una sensibilización y toma de conciencia con respecto a la sustantividad de que todos y todas seamos educados en la diversidad desde la equidad. La educación adquiere así un papel relevante, vinculado por esa finalidad básica de for- 
mación ciudadana del sistema educativo y la educación no formal con un sentido de responsabilidad política pública.

Así, insistimos en que pedagógicamente lo intercultural pretende promover relaciones dialógicas e igualitarias entre personas y grupos que pertenecen a mundos de significados distintos e interviene mediando en los conflictos presentes ineludiblemente en los procesos convivenciales de estos procesos, sin ignorar ni las relaciones de poder dadas, sino identificándolas y estableciendo recursos desde la formación cívica para la generación de estrategias que trabajen dichos conflictos. Además, no debemos olvidar que se trata de un proceso permanente, siempre inacabado, marcado por la deliberada intención de promover relaciones democráticas entre personas y grupos de identidades culturales distintas y no solamente una coexistencia pacífica, rompiendo con una visión esencialista de las culturas y de las identidades culturales y dotándolas de capacidad de apertura.

Frente a esto, una concepción de docentes y educandos con vocación intercultural debe llevar aparejada la inclinación hacia lo que podríamos denominar una actitud interculturalista (Gómez Lara, 2004) como componente ineludible de las competencias en clave intercultural. En este sentido, al hablar de competencias interculturales debemos referirnos a un conjunto de actitudes para desplegar ante la realidad del multiculturalismo social, conjugadas con valores éticos (igualdad, libertad, solidaridad, tolerancia, justicia...) y otras competencias basadas en los derechos humanos. De ahí que podamos justificar el interculturalismo y sus competencias como parte intrínseca a la pedagogía crítica y cuestiones ineludibles para abordar en la formación docente inicial y permanente. Desde esta óptica, y vinculado con el desarrollo teórico anterior, hay que considerar las siguientes competencias docentes, expresadas en términos de capacidades, como aspectos fundamentales para reflexionar y trabajar profesionalmente:

- Capacidad de análisis de otras culturas desde sus propios patrones culturales pero con el referente ético y crítico de los derechos humanos, de forma que no se dé paso a un relativismo cultural que puede acabar justificando prácticas culturales que atenten contra los derechos humanos y sí a una concepción de valoración y respeto a las diferencias.

- Capacidad de búsqueda del encuentro, lo que implica el ejercicio político que reniega de la etiqueta y la guetización al mismo tiempo que no teme el cambio inherente a la interacción, lo cual nos debe llevar también, por un lado, a entender ese encuentro como una oportunidad para el aprendizaje, y por otro, a desarrollar una concepción de la experiencia que se centre menos en el sujeto y más desde la confrontación con lo otro u otros en marcos culturales diversos (Innerarity, 2001). 
- Capacidad para el desarrollo de estrategias y tácticas para el trato y comprensión de lo diverso, lo que podríamos llamar 'traducción entre culturas' que nos ayudan a normalizar lo diferente transformándolo en una alteridad comprensible o al menos válida para la interlocución cultural.

- Capacidad para huir de la proyección como universal de un modelo cultural concreto, reflexionando sobre qué son exactamente las culturas de una sociedad multicultural y sobre el hecho de que toda acción educativa puede suponer, de algún modo, una forma de aculturación. Al fin y al cabo, pedagógicamente a menudo acabamos refiriéndonos a la cultura tal o cual como realidades de contenido estereotipado que asumimos constituyen a individuos que participan de una misma sociedad con otros diferentes. Por eso es necesario trascender las visiones estáticas y deterministas sobre las culturas, negando la presencia del otro u otra como algo definido de antemano por los demás otros, sobre todo si la definición proviene de la cultura hegemónica, así como evitar el uso instrumental de las culturas de referencia de las personas y sus identidades.

- Capacidad de fomento de la curiosidad epistemológica, siguiendo la terminología de Freire (1997) y diferenciándola de una curiosidad ingenua, sino entendiéndola en el ámbito intercultural como una curiosidad crítica, enriquecida con las teorías, lecturas, hipótesis y reflexiones que permite discernir (por tanto elegir) los elementos exteriores que potencian aún más las culturas de referencia e interacción. La curiosidad epistemológica crece, se retroalimenta a medida que cada vez conoce más sobre otras culturas, otros pensamientos.

- Capacidad de comprensión y desarrollo, dentro de un marco con vocación intercultural, de la solidaridad como forma de conocimiento que se obtiene por vía del reconocimiento del otro/a, que sólo puede ser reconocido/a en tanto que productor de conocimiento. La construcción de un conocimiento multicultural en esta línea tiene, según de Sousa Santos (2003), dos dificultades: el silencio y la diferencia. El primero, debido a la destrucción e infravaloración de muchas formas de saber que la ciencia moderna y el capitalismo salvaje han arrastrado a la invisibilidad e incluso la desaparición. La segunda, incide en que sólo existe conocimiento multicultural y, por tanto, solidaridad en las diferencias, pero estas diferencias deben ser inteligibles y ahí volvemos a la necesidad de la traducción inter-cultural.

- Capacidad de diálogo como referente permanente en la construcción de lo intercultural. Como dice Bilbeny $(2002,152)$ : «La sociedad es conversación, y cuanto más multicultural sea aquélla mejor deberá habituarse a prestar oídos a la diferencia en su interior y a entablar diálogo con ésta. (...) La interculturalidad nos hace ver de una vez que la 'ética del diálogo' es éti- 
ca en diálogo, y que el discurso dialógico necesita el apoyo de las virtudes dialogales. El modelo del diálogo nunca está concluido, sino que se encuentra haciéndose permanentemente, y lo que lo mantiene así es la escucha real de las perspectivas que entran en diálogo.»

- Capacidad empática, de forma que seamos capaces de identificar las vivencias del otro u otra e implicarnos en la búsqueda de alternativas desde la experiencia común en condiciones de equidad y desde la resolución pacífica de los conflictos inherentes a menudo desde la convivencia, sin dejar por ello de reconocer y valorar los contenidos transculturales que nos unen, de forma que «sólo así se podrán ir construyendo las necesarias zonas de contacto -de espacios culturales o zonas de mediación- que sirvan para materializar el resultado del encuentro entre las orillas, es decir, entre las diferentes y plurales formas de reaccionar culturalmente frente a la realidad» (Herrera, 2009, 81).

\section{A MODO DE CONCLUSIÓN} guiente:

Dice Amin Maalouf en su magnífico libro 'Identidades asesinas' lo si-

«En todas las épocas hubo gentes que nos hacen pensar que había entonces una sola pertenencia primordial, tan superior a las demás en todas las circunstancias que estaba justificado denominarla 'identidad'. La religión para unos, la nación o la clase social para otros. En la actualidad, sin embargo, basta con echar una mirada a los diferentes conflictos que se están produciendo en el mundo para advertir que no hay una única pertenencia que se imponga de manera absoluta sobre las demás. (...) si bien en todo momento hay, entre los componentes de la identidad de una persona, una determinada jerarquía, ésta no es inmutable, sino que cambia con el tiempo y modifica profundamente los comportamientos» (1999, 22-23).

El escritor libanés nos llama la atención con estas palabras sobre el carácter contingente, flexible, cambiante de las identidades y las diferencias y su importancia vital para nosotros y nosotras. Este carácter mutable e híbrido posee un potencial educativo ineludible, ya que nos hace percibir que, como toda dimensión humana, la experiencia personal y social se encuentra imbricada en la urdimbre de la cultura, en los múltiples y a menudo complejos mecanismos y significados que la configuran y en su propio arraigo desde realidades multiculturales como las actuales, con vocación intercultural. Por eso, tal y como planteaba Freire $(1997,50)$ : «La lucha ya no se reduce a retrasar lo que acontecerá o asegurar su llegada; es preciso reinventar el mundo. La educación es indispensable en esta reinvención. 
Considerarnos como sujetos y objetivos de la Historia nos hace seres de la decisión, de la ruptura. Seres éticos.»

Por eso resulta tan importante tanto el trabajo de conceptualización y aclaración de los significados aplicados al uso de las teorías, como es el caso de las relacionadas con la interculturalidad y las cuestiones identitarias y de diferencias, como ir visibilizando las competencias docentes emergentes de estas perspectivas teóricas. Así, teniendo en cuenta todo lo anterior, podemos considerar que partir del referente intercultural para pensar las diferencias y configurar competencias docentes debe llevarnos a conclusiones como las siguientes:

a) La necesidad de que la formación vehicule la práctica del respeto y valoración de los distintos grupos en interacción en el seno de la comunidad educativa, de forma que se promuevan actitudes positivas hacia los mismos y desde ellos a los demás, teniendo la labor docente una función importante de facilitación al respecto.

b) El desarrollo de estrategias para la gestión de la diversidad cultural y la contextualización de las propias concepciones docentes y creencias en la misma, de manera que nos permita trabajar sobre el fomento de las competencias interculturales en las y los demás y en nosotros mismos.

c) La búsqueda del sentido y el valor del encuentro como forma de abordar los conflictos interculturales e intentar desarrollar mecanismos pedagógicos de traducción para la convivencia sobre la base de los derechos humanos.

d) El fomento de la interrelación del pensar, hacer y sentir en la actividad educativa, de forma que se aborde la construcción de la ciudadanía intercultural tanto desde aspectos cognitivos y culturales como desde dimensiones más instrumentales y vinculadas con las competencias emocionales y una reflexión profunda en torno al mundo de los valores, para lo cual el educador/a debe tener una sólida formación en estas cuestiones.

e) La presencia en el diseño e implantación de acciones educativas vinculadas a la solidaridad, la tolerancia, la equidad y la justicia como aspectos sustanciales vinculados a las competencias interculturales.

f) El acceso a la vivencia y fomento de la ciudadanía intercultural desde el aprendizaje compartido y el sentido cooperativo de la prác- 
tica educativa, abarcando aspectos tales como la investigación relacionada con la educación intercultural, la innovación pedagógica, la formación en centros, instituciones y espacios educativos sobre estas cuestiones, el desarrollo de grupos de trabajo específicos, la creación colectiva de materiales didácticos interculturales para todo el alumnado, etc.

g) El conocimiento y generación de herramientas y espacios para la colaboración con el resto de la comunidad y otros agentes sociales y educativos (mediadores interculturales, servicios sociales, centros de salud, etc.), logrando la apertura de la escuela al contexto inmediato y coordinando las actuaciones necesarias para fomentar la sensibilización y el desarrollo cívico intercultural en la comunidad. 


\section{REFERENCIAS BIBLIOGRÁFICAS}

Bauman, Z. (2007). Confianza y temor en la ciudad. Vivir con extranjeros. Barcelona: Arcadia.

Bilbeny, N. (2002). Por una causa común. Ética para la diversidad. Barcelona: Gedisa.

Bruner, J. (1997). La educación, puerta de la cultura. Madrid: Visor.

Freire, P. (1997). A la sombra de este árbol. Barcelona: El Roure.

García Canclini, N. (2005). Diferentes, desiguales y desconectados. Mapas de la interculturalidad. Barcelona: Gedisa.

García, J. A. y Goenechea, C. (2009). Educación intercultural. Análisis de la situación y propuestas de mejora. Madrid: Wolters Kluwer.

Giroux, H. (2001). Cultura, política y práctica educativa. Barcelona: Graó.

Gómez Lara, J. (ed.) (2004). La escuela intercultural: regulación de conflictos en contextos multiculturales. Madrid: La Catarata.

Herrera, J. (2009). Derechos Humanos, interculturalidad y racionalidad de resistencia, en Muñoz, A. y Molina, B. (ed.) Pax Orbis. Complejidad y conflictividad de la paz. Granada: Editorial Universidad de Granada.

Innerarity, D. (2001). Ética de la hospitalidad. Madrid: Península.

Maalouf, A. (1999). Identidades asesinas. Madrid: Alianza.
Marí Ytarte, R. (2007). Cultura y diversidad. Pensar la intercultura en educación. Barcelona: Gedisa

Martínez de Bringas, A. (2007). Interculturalidad en tiempos globales: el reto de los derechos de las mujeres migrantes, en Vila Merino, E. S. (Coord.) Pedagogía de la alteridad. Interculturalidad, género y educación. Madrid: Popular, 27 - 42.

Pérez Tapias, J.A. (2007). Del bienestar a la justicia, Aportaciones para una ciudadanía intercultural. Madrid: Trotta.

Sousa Santos, B. (2003). Crítica de la razón indolente. Bilbao: Desclée de Brower.

Touriñán, J.M. (2008). El reto de la educación intercultural como ejercicio de educación en valores, en Toruiñán, J.M. (ed.) Educación en valores, educación intercultural y formación para la convivencia pacífica. A Coruña: Netbiblo.

Vila Merino, E. S. (2007). Pedagogía de la alteridad. Interculturalidad, género y educación. Madrid: Popular.

Zizëk, S. (1998). Multiculturalismo o la lógica cultural del capitalismo multinacional, en Jameson, F. y Zizëk, S. (comps.) Estudios culturales. Reflexiones sobre le multiculturalismo. Buenos Aires: Paidós. 


\section{PALABRAS CLAVE}

Educación intercultural, Pedagogía intercultural, Identidad, Diferencia, Multiculturalismo, Competencias educativas.

\section{KEY WORDS}

Intercultural education, Intercultural pedagogy, Identity, Difference, Multiculturalism, Educational Competences.

\section{PERFIL ACADÉMICO Y PROFESIONAL DEL AUTOR}

Prof. Dr. Eduardo S. Vila Merino, ha sido Maestro de Educación Primaria y actualmente Profesor Contratado Doctor en el Dpto. de Teoría e Historia de la Educación de la Universidad de Málaga (España).

Siete libros publicados. Diez capítulos de libro. Una veintena de artículos publicados en revistas nacionales e internacionales. Ponencias y comunicaciones en más de treinta congresos y jornadas. Estancias en varias universidades latinoamericanas (Argentina, Venezuela, Honduras, México). Principales líneas de investigación: teoría de la educación, educación intercultural, coeducación, derechos humanos, política educativa.

Dirección del Autor: Departamento de Teoría e Historia de la Educación Facultad de Ciencias de la Educación Universidad de Málaga Campus de Teatinos, s/n (29076) Málaga, España E-mail: eduardo@uma.es

Fecha Recepción del Artículo: 23. Agosto. 2010

Fecha Revisión del Artículo: 12. Enero. 2011

Fecha Aceptación del Artículo: 23. Febrero. 2011

Fecha de Revisión para publicación: 15. Noviembre. 2011 
